\title{
Evidence of the $\gamma$ Doradus nature for a group of candidates
}

\author{
S. Martín, M. Bossi, and F. M. Zerbi
}

\author{
INAF-Osservatorio Astronomico di Brera, via Bianchi 46, 23807 Merate (LC), Italy \\ e-mail: bossi@merate.mi.astro.it; zerbi@merate.mi.astro.it
}

Received 16 October 2002 / Accepted 31 January 2003

\begin{abstract}
We present in this paper new $u v b y \beta$ photometry of eight stars suspected to be $\gamma$ Doradus variables according to Handler \& Kaye (2000). The new observational material has been analyzed and previously published data re-analyzed by means of an objective period search technique, based on the least-squares algorithm, called Multifre (Bossi \& Nuñez 2002). We found evidence for the inclusion of seven of these stars in the list of recognized $\gamma$ Doradus variables.
\end{abstract}

Key words. stars: variables: general - stars: oscillations - stars: fundamental parameters - techniques: photometric

\section{Introduction}

The $\gamma$ Doradus group has been recently defined by Kaye et al. 1999 as a class of low amplitude high $n$ low $l$ non-radial $g$-mode pulsating stars, lying on or just beyond the cold border of Cepheid Instability strip. The typical periods of pulsation in these stars range between 0.3 and 3 days and the typical amplitude ranges between the limit of detection and a tenth of a magnitude. Some of the known candidates show up to six frequencies simultaneously excited.

The characteristics mentioned above could be worked out in spite of the limited number of confirmed $\gamma$ Doradus known at the date, a couple of dozens. Although their general physical characteristics are very homogeneous each of them has its own pattern and peculiarity. Beyond some peculiarities (reviewed e.g. by Zerbi 2000), the $\gamma$ Dor class shows a remarkable physical homogeneity: these stars occupy a very small area in the HR diagram and all their metallicities are around solar values. This observational evidence indicates that the $\gamma$ Dor phenomenon occurs only under well-defined conditions.

In the last three years, an intensive search for these variables has been made with the purpose of discovering a larger number of them and hence figuring out the origin of their pulsation and its relationship with their position in the HR diagram. Theoretical work on these variables has progressed at the same pace. Guzik et al. (2000) proposed recently the first specific model accounting for $\gamma$ Doradus pulsation. This model is interesting because it attributes to the convection layer and associated magnetic fields a crucial role in determining the pulsational characteristics in these stars.

Because of their photometric behaviour (long periods and low amplitudes), the detection of these variables is not an easy task. Their discovery among field stars is almost only

Send offprint requests to: S. Martín,

e-mail: susana@merate.mi .astro.it achievable serendipitously and, once discovered, they need multi-longitude observing campaigns to conveniently sample their curves. Examples are the works published by Balona et al. (1996), Breger et al. (1997) and Zerbi et al. (1999).

Open clusters, as collections of stars with observational homogeneous characteristics, offer the possibility to perform systematic surveys in search for these stars as done by Martín \& Rodríguez (2000). The open cluster environment provides us also useful additional information about the physical parameters of the stars and their possible role in originating pulsation of the $\gamma$ Dor type. As a matter of fact, a high number of $\gamma$ Doradus candidates belongs to open clusters (see Zerbi et al. 1998; Kim et al. 2001; Arentoft et al. 2001; Martín 2002), whether this is a sample bias or not is not known yet.

In addition to the open clusters, the Hipparcos Catalogue (ESA 1997) has been perhaps the major source of $\gamma$ Doradus candidates so far. Two systematic browsing works have been done on Hipparcos data by Aerts et al. (1998) and later by Handler (1999), both with the purpose to point out possible candidates: the two works reported 14 and 70 new possible candidates respectively. Anyway, any detection based on the Hipparcos data has to be confirmed. Indeed the photometric quality of the Hipparcos light curve is poor and a color information, needed to state the intrinsic character of variability, is not available. Some of these candidates have then been monitored more intensively with the purpose to confirm their nature. It is the case of eleven F0-F9 stars contained both in the Geneva photometric database and the Hipparcos Catalogue. Eyer \& Aerts (2000) monitored these stars confirming the $\gamma$ Doradus nature for three of them.

In this stream, we decided to perform a photometric observational campaign dedicated to eight stars selected in the Catalogue of $\gamma$ Doradus Stars (Handler \& Kaye 2000; April 2000 version) where they are marked as candidates to be confirmed. Beside the analysis of our new data, we performed 
an extensive reanalysis of previously published light curves by means of a new period-search code (Bossi \& Nuñez 2002).

In addition, no doubt the era of space asteroseismology will add interest to the role of the $\gamma$ Doradus pulsating mechanism in bridging the gap between the overstable $\delta$ Scuti pulsations and the oscillations stochastically generated in the convection layers of solar type stars (see Zerbi \& Kaye 2002). In fact, photometric tools such as COROT (Baglin \& Foing 1999), MONS (Kjeldsen et al. 2000) and MOST (Matthews et al. 2000), will be likely to allow us to enlarge our observational sample of solar type light curves (which is currently restricted to the Sun) and to detect possible faint signals of $p$ mode pulsations shocked by thin convective layers in $\gamma$ Doradus stars.

\section{Observations and data reduction}

The unpublished observations presented in this paper were collected during a campaign in January 1-14, 2001 using the $90 \mathrm{~cm}$ telescope at Observatorio de Sierra Nevada, Spain. The photometer attached to this telescope is a six-channel $u v b y \beta$ spectro-photometer, with non-cooled EMI photomultipliers of the type 9789 QA. Because of the bad weather conditions, only five of the fourteen nights were suitable for observation. The measurements were collected using the simultaneous uvby filters while observations in $\mathrm{H}_{\beta}$ were performed only in those cases in which $\beta$ index were not available in literature.

The candidate stars have been selected among the $\gamma$ Doradus listed by Handler \& Kaye (2000, Table 3). The main criterion of selection was the visibility during the campaign: $5^{\mathrm{h}} 00<\alpha<9^{\mathrm{h}} 30^{\prime}$ and $\delta>-10^{\circ}$. The sample was limited to 10 stars to allow us to collect a sufficient number of measurement per star and per night. Two of these stars, HD 33204 and HD 35187, members of visual multiple systems (CCDM J05098+2802A and CCDM J05240+2458A), were not included in our final analysis. Given that the angular separations of both binary systems are 11.8 and 1.3 arcsec respectively, the available diaphragms did not allow a reliable flux separation between targets and their corresponding companion stars.

For each observed object two comparison stars, main $\left(C_{\mathrm{m}}\right)$ and check $\left(C_{\mathrm{ck}}\right)$, were chosen. A sequence of three cycles (Sky$C_{\mathrm{m}}-\mathrm{Star}_{i}-C_{\mathrm{ck}}$ ) was adopted for each star and the order of execution of these cycles optimized for telescope motion, i.e. ordered in function of the position of the star in the sky. We also kept in mind to maintain the homogeneity in the data set as high as possible. The number of background measurements in the observing sequence was adjusted to the position and phase of the Moon.

In order to reach an internal error of less than 0.002 per observation in the $v$ and $b$ bands, we selected an integration time of 30,35, $40 \mathrm{~s}$ for stars with magnitude 6,7 and 8 respectively. All the selected program, comparison and check stars fall in the above magnitude range. We have then used the procedure described in Rodríguez (1997) to transform our data into the standard $u v b y \beta$ system.

A complete list of program stars together with the corresponding main comparison and check stars is reported in Table 1. We show in the same table the number of nights and the number of measurements collected and the signal-to-noise ratios in the filters $v$ and $b$ for each program and check star. The white noise contained in each time series has been evaluated from the root-mean-square difference between closely consecutive data. The corresponding signal-to-noise ratio, obtained assuming this white noise value as representative of the whole noise, is given in magnitudes (positive values correspond to very noisy signals).

\section{Physical parameters}

The physical parameters of the eight candidate stars published in the Hipparcos Catalogue are listed in Table 2. For each star we present: the Hipparcos identifier, the $V$ magnitude, the $B-V$ colour index, the absolute visual magnitude $M_{\mathrm{V}}$, the spectral type and the trigonometric parallax. The same table indicates the number of measurements and the related $S / N$ ratio for each star in the program derived using the method described above.

On the other hand, Table 3 summarizes the $u v b y \beta$ indices for all the candidates stars as well as those physical parameters computed via the Strömgren photometry. These indices are those found in Hauck \& Mermilliod (1998) except for the two stars without previously published $\mathrm{H} \beta$ photometry, HD 48271 and HD 69715, for which the indices have been calculated via the present data with standard errors of 0.008 and 0.006 respectively. Out of the 16 comparison stars adopted, nine do not have published $u v b y \beta$ photometry. So, as extra results of our observations, Strömgren indices have been computed using the present observations and reported in Table 4.

We computed the de-reddened indices using the method described in Philip et al. (1976) and the reference lines of Philip \& Egret (1980). The metallicities $[\mathrm{Me} / \mathrm{H}]$ are derived using the Nissen (1988) and Smalley (1993) relations for type-AFG stars while surface gravities $\log (g)$ and temperatures $T_{\text {eff }}$ have been determined using the grids $([\mathrm{Me} / \mathrm{H}]=0.00)$ given by Smalley \& Kupka (1997). No values of rotational velocities have been found in the bibliography except for HD 49015 (Nordström 1997) and HD 81421 (Handler \& Kaye 2000) with 44 and $19 \mathrm{~km} \mathrm{~s}^{-1}$, respectively. We notice that calculated values for all the programmed stars are very close to the typical ones for $\gamma$ Dor stars: $<T_{\text {eff }}>=7160 \mathrm{~K},<[\mathrm{Me} / \mathrm{H}]>=-0.06$ dex and $<M_{\mathrm{V}}>=2$. 69 (Kaye et al. 1999).

One of the stars, HD 81421, is an X-ray source with $L_{\mathrm{x}}=10^{28} \mathrm{erg} \mathrm{s}^{-1}$ catalogued in the EMSS (Einstein Extended Medium Sensitivity Survey) and confirmed by ROSAT observations (Fleming et al. 1995). Micela et al. (1997) analyzed the sub-sample obtained cross-correlating the Hipparcos Input Catalogue (HIC, Turon et al. 1992) and the EMSS Catalogue with the purpose of determining the characteristics of an X-ray selected sample of stars in the solar neighbourhood: HD 81421 was included in their sample. The most important conclusion relevant to the $\gamma$ Doradus phenomenon drawn by the authors is that most of the stars in their sample are young (Pleiades age), active ZAMS stars.

We mention this because age and activity are important issues in $\gamma$ Dor stars. Indeed the model presented in Guzik et al. (2000) and previously depicted by Kaye \& Zerbi (1997) is based on the presence of magnetic fields in the thin convection 
Table 1. Complete $\log$ of the new observations presented in this paper. For each star we report the name in the first column, the name of the comparison stars in the second and third column and the number of observing night in the fourth column. We then report the number of measurements and the $S / N$ of the variable-comparison differential $v$ and $b$ time series in the fifth, sixth and seventh column. Equivalent quantities for the check-comparison $v$ and $b$ time series are reported in the eighth, ninth and tenth column. See the text for a detailed description of the $S / N$ ratio calculation.

\begin{tabular}{rcccccccrr}
\hline \hline $\begin{array}{c}\text { Star } \\
(\mathrm{HD})\end{array}$ & $\begin{array}{c}C_{\mathrm{m}} \\
(\mathrm{HD})\end{array}$ & $\begin{array}{c}C_{\mathrm{ck}} \\
(\mathrm{HD})\end{array}$ & nights & $N_{\mathrm{st}}$ & $\begin{array}{c}S / N_{\mathrm{st}}(v) \\
(\mathrm{mag})\end{array}$ & $\begin{array}{c}S / N_{\mathrm{st}}(b) \\
(\mathrm{mag})\end{array}$ & $N_{\mathrm{ck}}$ & $\begin{array}{c}S / N_{\mathrm{ck}}(v) \\
(\mathrm{mag})\end{array}$ & $\begin{array}{c}S / N_{\mathrm{ck}}(b) \\
(\mathrm{mag})\end{array}$ \\
\hline 48271 & 48272 & 49199 & 4 & 62 & -3.7 & -3.7 & 55 & -0.3 & 0.8 \\
49015 & 48977 & 49245 & 4 & 52 & -1.5 & -1.7 & 52 & -1.4 & -1.5 \\
63436 & 63162 & 63957 & 5 & 49 & -4.4 & -2.4 & 46 & -0.2 & -2.0 \\
65526 & 65877 & 65409 & 5 & 46 & -3.6 & -3.1 & 43 & -0.6 & 2.1 \\
69715 & 69961 & 69330 & 5 & 57 & -3.6 & -3.5 & 56 & -0.8 & -1.3 \\
70645 & 71233 & 69892 & 5 & 54 & -5.1 & -5.9 & 51 & 0.9 & -0.2 \\
80731 & 80042 & 81807 & 5 & 50 & -3.4 & -3.7 & 49 & -0.5 & -0.6 \\
81421 & 80917 & 81464 & 4 & 47 & -4.2 & -3.3 & 46 & 1.5 & 1.2 \\
\hline
\end{tabular}

Table 2. Physical parameters and number of measurements for the each programmed star taken from Hipparcos Catalogue. The corresponding $S / N$ values are showed in the last column.

\begin{tabular}{cccccccrr}
\hline \hline HD & HIP & ST & $\begin{array}{c}V \\
(\mathrm{mag})\end{array}$ & $\begin{array}{c}B-V \\
(\mathrm{mag})\end{array}$ & $\begin{array}{c}M_{\mathrm{V}} \\
(\mathrm{mag})\end{array}$ & $\begin{array}{c}\pi \\
(\mathrm{mas})\end{array}$ & $\begin{array}{r}N_{\text {Hip }} \\
\begin{array}{c}S / N_{\text {Hip }} \\
(\mathrm{mag})\end{array}\end{array}$ \\
\hline 48271 & 32263 & F0 & 7.49 & 0.315 & 2.62 & 10.62 & 120 & -1.1 \\
49015 & 32475 & F0IV & 7.04 & 0.375 & 2.83 & 14.37 & 126 & 1.3 \\
63436 & 38138 & F2 & 7.46 & 0.356 & 3.52 & 16.26 & 54 & -1.2 \\
65526 & 39017 & A3 & 6.98 & 0.297 & 2.85 & 16.20 & 100 & -1.8 \\
69715 & 40791 & A5 & 7.18 & 0.360 & 3.09 & 15.19 & 105 & -0.1 \\
70645 & 41488 & F0 & 8.12 & 0.344 & 2.51 & 7.55 & 364 & 2.4 \\
80731 & 46099 & F0V & 8.46 & 0.345 & 2.61 & 6.77 & 309 & -0.8 \\
81421 & 46233 & A3 & 7.01 & 0.280 & 2.79 & 14.29 & 116 & -1.1 \\
\hline
\end{tabular}

layer of these stars. The role of the field is to freeze the plasma and allow the pulsation generated below the convection layer to emerge at the surface not being disrupted, as would normally happen, by the convection itself. The HR area inhabited by the $\gamma$ Dor class coincides with the locus where magnetic breaking occurs (Fukuda 1982). Young stars in the area are expected to have appreciable magnetic fields while older stars should have completed the breaking process and exhibit slow rotation and marginal magnetic activity. An indication of youth and activity for the stars in the sample goes in the direction of confirming the scenario of the presently available models.

The positions of the observed stars in the HR diagram is shown in Fig. 1. The ZAMS line was drawn from Philip \& Egret (1980) and the observational edges of the $\delta$ Scuti instability strip are from Rodríguez \& Breger (2001). All the stars considered in this paper lie into the well-defined domain of $\gamma$ Doradus variables, i.e., on the intersection between the main sequence and the cool border of Cepheid instability strip.

\section{Frequency analysis}

For each of the stars in the program we analyzed the new Sierra Nevada (OSN) data set and re-analyzed the Hipparcos light curves by means of the Multifre code (Bossi \& Nuñez 2002).

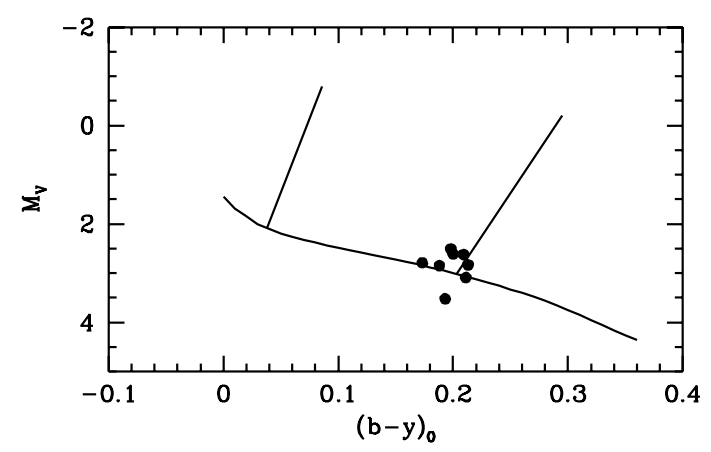

Fig. 1. Positions of the candidates in the HR diagram. The $M_{\mathrm{V}}$ values are from Hipparcos Catalogue while the $(b-y)_{0}$ indices have been calculated for this paper.

The Multifre code searches for the least squares best fit of a time series using a finite number of sinusoids under the only assumption that the best fitting set of $n$ frequencies contains as a subset, except for a non-linear fine adjustment, at least one of the $N$ best fitting sets of $n-1$ frequencies, where $N$ is a parameter controlled by the user (we set $N=50$ ).

In most of the codes used for sinusoidal period search, either based on periodograms or least squares methods, the selection of the significant frequency is done by the operator by 
Table 3. $u v b y \beta$ indices and calculated physical parameters.

\begin{tabular}{lccccccc}
\hline \hline HD & $\begin{array}{c}\beta \\
(\mathrm{mag})\end{array}$ & $\begin{array}{c}b-y \\
(\mathrm{mag})\end{array}$ & $\begin{array}{c}m_{1} \\
(\mathrm{mag})\end{array}$ & $\begin{array}{c}c_{1} \\
(\mathrm{mag})\end{array}$ & $\begin{array}{c}T_{\text {eff }} \\
(\mathrm{K})\end{array}$ & $\log g$ & $\begin{array}{c}{[\mathrm{Me} / \mathrm{H}]} \\
(\mathrm{dex})\end{array}$ \\
\hline 48271 & 2.735 & 0.210 & 0.163 & 0.634 & 7060 & 4.27 & -0.10 \\
49015 & 2.726 & 0.222 & 0.175 & 0.645 & 7020 & 4.20 & 0.08 \\
63436 & 2.756 & 0.206 & 0.172 & 0.631 & 7230 & 4.45 & -0.04 \\
65526 & 2.734 & 0.188 & 0.151 & 0.662 & 7240 & 4.35 & -0.29 \\
69715 & 2.729 & 0.211 & 0.157 & 0.652 & 7030 & 4.18 & -0.15 \\
70645 & 2.706 & 0.198 & 0.160 & 0.652 & 7150 & 4.30 & -0.21 \\
80731 & 2.723 & 0.200 & 0.160 & 0.693 & 7100 & 4.12 & -0.14 \\
81421 & 2.751 & 0.173 & 0.169 & 0.754 & 7310 & 4.13 & -0.13 \\
\hline
\end{tabular}

picking up the highest peak in the spectrum. When low $S / N$ signal is expected or where multiple frequencies are present, such a selection might be a misinterpretation that propagates into to final solution. Indeed the correspondent prewhitening (in the case of periodograms) or its insertion as known constituent (in the case of least square techniques) alters accordingly the successive mathematical problem. Multifre does not rely on a human decision but it considers the $N$ possibilities of the $N$ highest peaks in each successive spectrum. Moreover the successive spectral analysis is not stopped by human judgment at a given number of frequencies but is stopped when the residuals reach the level of the white noise.

In all the preliminary tests performed by the co-writers, this approach proved more effective than the other available methods in finding out the best mathematical model for the data. Nevertheless, since mathematics is not physics, in the presence of not optimal spectral windows and/or of low signal-to-noise ratios, the frequency set relevant to the physical problem may hid in a wood of fitting sets which are substantially equivalent from the point of view of the residual dispersion. In these cases however, traditional methods based on the human selection of frequencies does not provide more reliability since power spectra are generally crowed and dirty.

The substantial improvement represented by Multifre with respect to traditional methods in the reduction of non-optimal data set is that it provides a set of mathematical solutions. Other results can then be used to pick up the physical solution from this set, i.e. the simultaneous presence of a set or a sub-set of frequencies in data collected independently.

The data analyzed in this paper are a good example of how Multifre provide reliable solutions. Indeed both data sets are not optimal for a low amplitude frequency search by means of traditional methods. Due to weather conditions a low number of data points could be collected in the limited temporal baseline of the ONS observing campaign. They were however of high photometric precision. On the other hand, Hipparcos light curves have a large number of data points distributed in a long baseline but of poor photometric quality. The relevant point is that we have two absolutely independent data set: the presence in both time-series of a set or sub-set of frequencies has to be regarded as a strong evidence of physical significance for such set. Even more if each set of frequencies has been derived with an objective period search technique such as Multifre.
Table 4. $V$ and $u v b y$ indices corresponding to the comparison stars whose values non-existing to date.

\begin{tabular}{crrrr}
\hline \hline HD & $\begin{array}{c}V \\
(\mathrm{mag})\end{array}$ & $\begin{array}{c}b-y \\
(\mathrm{mag})\end{array}$ & $\begin{array}{c}m_{1} \\
(\mathrm{mag})\end{array}$ & $\begin{array}{c}c_{1} \\
(\mathrm{mag})\end{array}$ \\
\hline 49199 & 7.986 & 0.083 & 0.172 & 1.038 \\
& 5 & 5 & 5 & 12 \\
49245 & 7.684 & 0.018 & 0.157 & 1.128 \\
& 7 & 3 & 3 & 8 \\
63957 & 8.243 & 0.070 & 0.190 & 1.181 \\
& 7 & 5 & 5 & 15 \\
65409 & 8.110 & 0.022 & 0.090 & 0.563 \\
& 26 & 10 & 10 & 31 \\
65877 & 8.053 & 0.011 & 0.123 & 0.945 \\
& 21 & 6 & 4 & 16 \\
69961 & 7.146 & 0.096 & 0.188 & 0.980 \\
& 4 & 4 & 4 & 7 \\
71233 & 7.166 & 0.031 & 0.113 & 1.098 \\
& 5 & 3 & 4 & 8 \\
80917 & 7.325 & 0.130 & 0.190 & 0.859 \\
& 4 & 3 & 4 & 10 \\
81807 & 9.139 & 0.290 & 0.140 & 0.456 \\
& 7 & 6 & 7 & 19 \\
\hline
\end{tabular}

For the OSN data, the $v$ and $b$ curves were used in the frequency analysis independently. In both Strömgren and Hipparcos data sets the Multifre code stopped once reached the level of the white noise. In those cases where the $v$ and $b$ measurements gave a different solution, the analysis has been stopped at the last coherent passage.

The best frequency fitting proposed by Multifre at each successive step for both Hipparcos and Sierra Nevada data sets is listed in Table 5. Also we reported in Cols. 9-11 the frequency estimates published by other authors: (A) Hipparcos Catalogue, (B) Handler (1999) and (C) others, being Henry \& Henry (2000) the single additional source where an analysis of an different data set has been performed. To compare the results obtained, different fonts with different meaning have been used. Frequency values confirming within the error bars those obtained by other authors have been marked in bold. Frequency values found in both Hipparcos and the OSN data sets have instead been marked in italic. Values found in both data sets and confirming those given in literature are marked in italic-bold. Any of the above levels of coherence has been considered as a sign of physical meaning of that specific frequency.

We present in Fig. 2 the phased diagrams for the Hipparcos and the OSN measurements in the $V$ and $v$ filters respectively. In the case of the Hipparcos photometry, the curve for each component is phased after subtraction of all the other known frequencies obtained in the last corresponding frequency model. Regarding the OSN data, we have plotted those components more significant for each star allowing us to visually appreciate the physical significance of the proposed solutions. For those who do not trust visual examination of a phased curve a diagnostic for the presence of a frequency we propose the synoptic representation of the contribution of each frequency model to the reduction of the dispersion for both photometries in Fig. 3. This figure is a representation of the 
Table 5. Result of the frequency analysis for the program stars. Values obtained via Multifre for the Hipparcos curves are reported in the first column while values for OSN data in the second column. Each successive model is reported per increasing number of components. The third column reports the data available in literature which are taken from (A) Hipparcos Catalogue (ESA 1997), (B) Handler (1999) or (C) Others, where only the frequency taken from Henry \& Henry (2000) is found. A semi-column beside a frequency value has the same meaning than in Handler (1999), i.e., average values of several possible further frequencies. The label "Unsolved var" is according to the classification given by Hipparcos data base (see Variability Annex) (ESA 1997).

\begin{tabular}{|c|c|c|c|c|c|c|c|c|c|c|}
\hline Program Star & 1 & 2 & 3 & 4 & 5 & 1 & 2 & $\mathrm{~A}$ & $\mathrm{~B}$ & $\mathrm{C}$ \\
\hline \multirow[t]{4}{*}{ HD 48271} & 0.52436 & 0.54463 & 0.86914 & 0.86922 & & 0.918 & 0.623 & 0.5244 & 0.524 & \\
\hline & & 0.87183 & 0.90716 & 0.90722 & & & 0.750 & 0.8691 & 0.869 & \\
\hline & & & 0.91776 & 0.91786 & & & & & & \\
\hline & & & & 1.78336 & & & & & & \\
\hline HD 49015 & 2.20495 & & & & & & & & & 2.8969 \\
\hline \multirow[t]{2}{*}{ HD 63436} & 0.85434 & 0.85431 & & & & 1.435 & 1.402 & Unsolved & 1.4: & \\
\hline & & 1.40672 & & & & & 1.404 & & & \\
\hline \multirow[t]{4}{*}{ HD 65526} & 1.55287 & 1.55282 & 1.16572 & 0.37519 & & 1.556 & & 0.77641 & 1.553 & \\
\hline & & 1.67325 & 1.55275 & 1.16572 & & & & & 1.672 & \\
\hline & & & 1.67329 & 1.55277 & & & & & & \\
\hline & & & & 1.67322 & & & & & & \\
\hline \multirow[t]{4}{*}{ HD 69715} & 2.36461 & 2.01289 & 1.77841 & 1.77836 & & 2.575 & 1.728 & Unsolved & 2.364 & \\
\hline & & 2.36462 & 2.59878 & 2.59871 & & & 2.425 & & 2.0: & \\
\hline & & & 2.82043 & 2.82041 & & & & & & \\
\hline & & & & 3.08242 & & & & & & \\
\hline \multirow[t]{2}{*}{ HD 70645} & 1.26180 & 0.77076 & & & & 0.877 & 0.871 & 1.21230 & 1.212 & \\
\hline & & 1.26186 & & & & & 1.449 & & $0.8:$ & \\
\hline \multirow[t]{5}{*}{ HD 80731} & 0.89639 & 0.89636 & 0.89638 & 0.78203 & 0.01488 & 0.143 & 0.447 & 0.89641 & 0.896 & \\
\hline & & 1.34160 & 1.34168 & 0.79847 & 0.23316 & & 0.713 & & $0.7:$ & \\
\hline & & & 1.35642 & 0.89635 & 0.89638 & & & & & \\
\hline & & & & 0.98191 & 1.34168 & & & & & \\
\hline & & & & & 1.35642 & & & & & \\
\hline HD 81421 & 2.04193 & & & & & 2.046 & & 2.041966 & & \\
\hline
\end{tabular}

photometric quality and of the multiperiodic behaviour of these stars. In general, both types of lines corresponding to each data set are located in a well-defined region inside panel. For the stars HD 63436 and HD 65526, to consider a single frequency as solution from our OSN photometry is not enough to reach the white noise. Consequently, to explain such residuals more than one component would be necessary, or the signal could be quasi-periodic.

\section{Discussion}

All the stars in our program have been observed by Hipparcos satellite and, with the exception of HD 49015, the Hipparcos Catalogue reports these stars as photometrically variable: the variability of HD 49015 has been instead discovered by Henry \& Henry (2000). With the further exception of HD 81421, the program stars were included in the list of possible $\gamma$ Dor stars obtained via an independent re-analysis of Hipparcos light curves by Handler (1999).

The analysis presented in this paper has the purpose to confirm the suspected $\gamma$ Doradus nature of the program stars taking into account the results obtained previously. In this sense we first have to confirm that each of the program stars is effectively a variable. This can be done via the comparison between the $S / N$ of each of the program stars with respect to the estimated white noise and with the $S / N$ of a constant star observed under the same conditions.

As regards OSN data we notice that the $S / N$ values in both $v$ and $b$ filters reported in Table 3 are well above both the levels of the white noise and the $S / N$ of the comparison stars, clearly indicating photometric variability. HD 49015 is the only exception. However, if we compare these quantities with those listed in the last column in Table 2, i.e., the intrinsic $S / N$ of the Hipparcos curves of each star, we find lower values which would not, by themselves, be representative of variable curves. Such lower values are likely due to the poor accuracy of the Hipparcos photometry, or to an under-estimate of the $S / N$ due to the lack of near enough measurements in the sampling of the Hipparcos curves. A clear example is the $S / N$ of the HD 70645 (2.4 mag) where, as we will describe later on, no variability was detected before data cleaning. Even data cleaning could not explain the low value (1.3 mag) of HD 49015, the variability of which could not be confirmed.

As a second step, we have to establish which of the stars found to be variables show multiperiodic variability. Multiperiodicity, together with the physical parameter reported in Sect. 3, can be considered as a signature of the belonging to the $\gamma$ Dor class. Indeed no other multiperiodic variables 


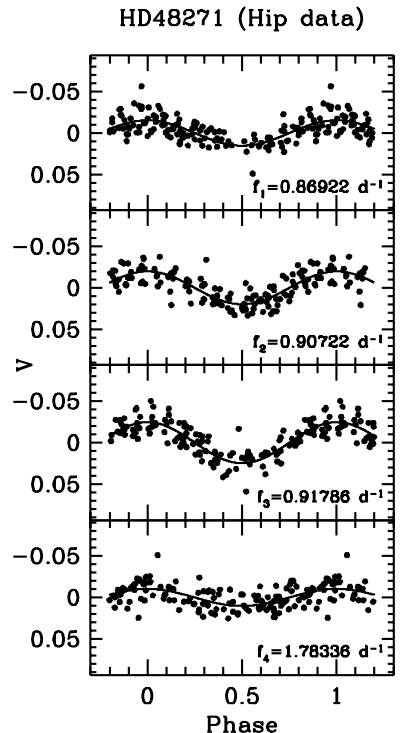

HD48271 (OSN data)
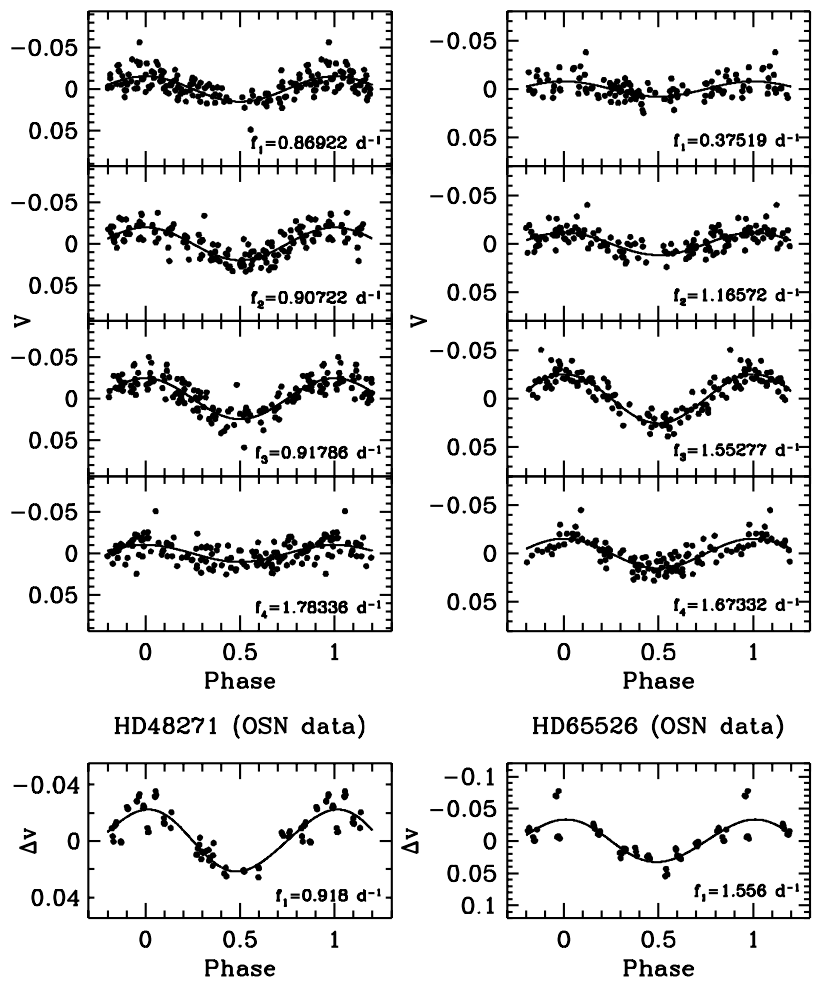

HD65526 (OSN data)
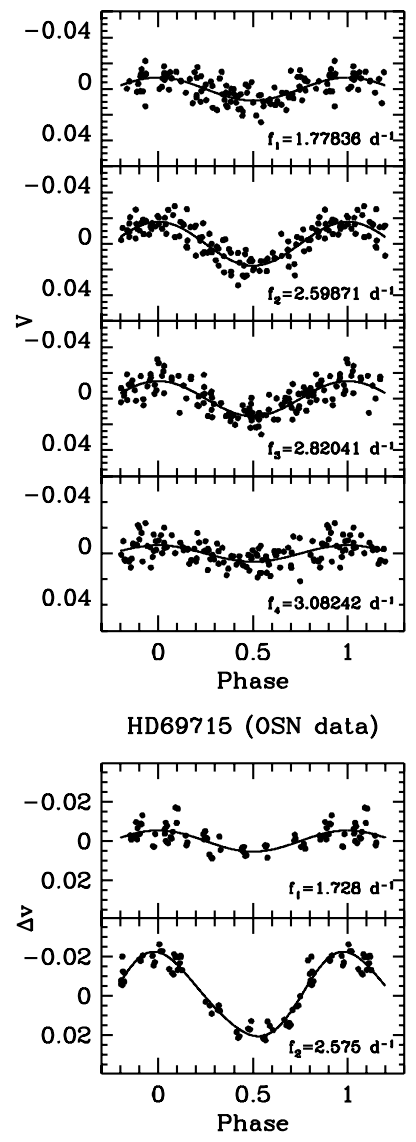

HD69715 (OSN data)

$z_{-0.05}^{0.05}$

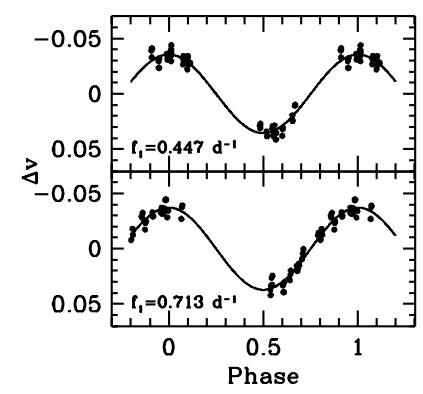

HD 49015 (Hip data)
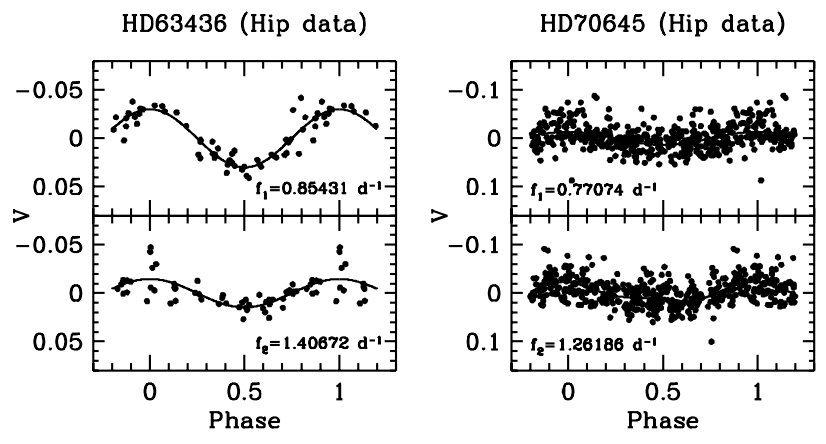

HD63436 (OSN data)

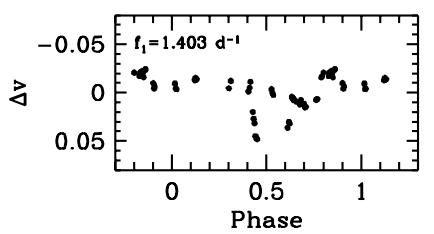

HD 80731 (Hip data)

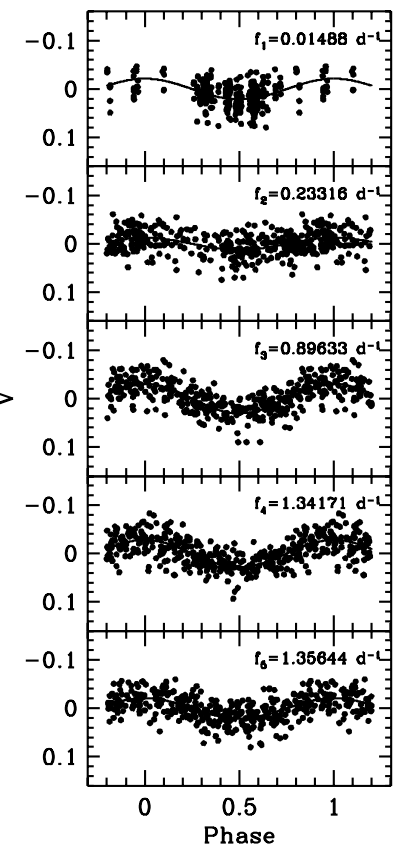

HD 80731 (OSN data)

HD81421 (Hip data)

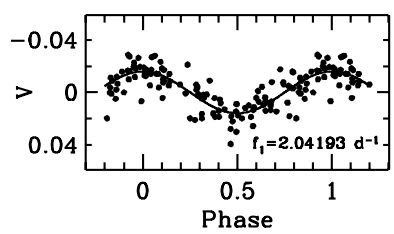

HD81421 (OSN data)

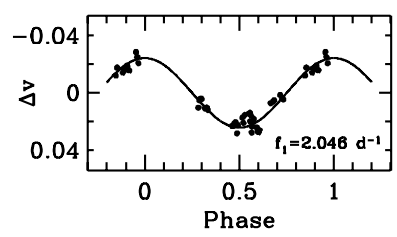

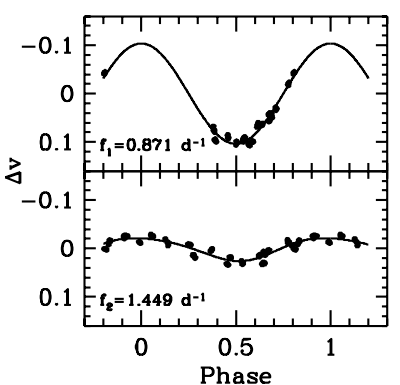

Fig. 2. Phase diagrams on the V Hipparcos (top panels) and $v$ OSN (bottom panels) data sets for all stars. The plots were computed with the same values of the frequencies represented in Fig. 3 while the curves are phased with respected to different $T_{0}$ for each data set. 

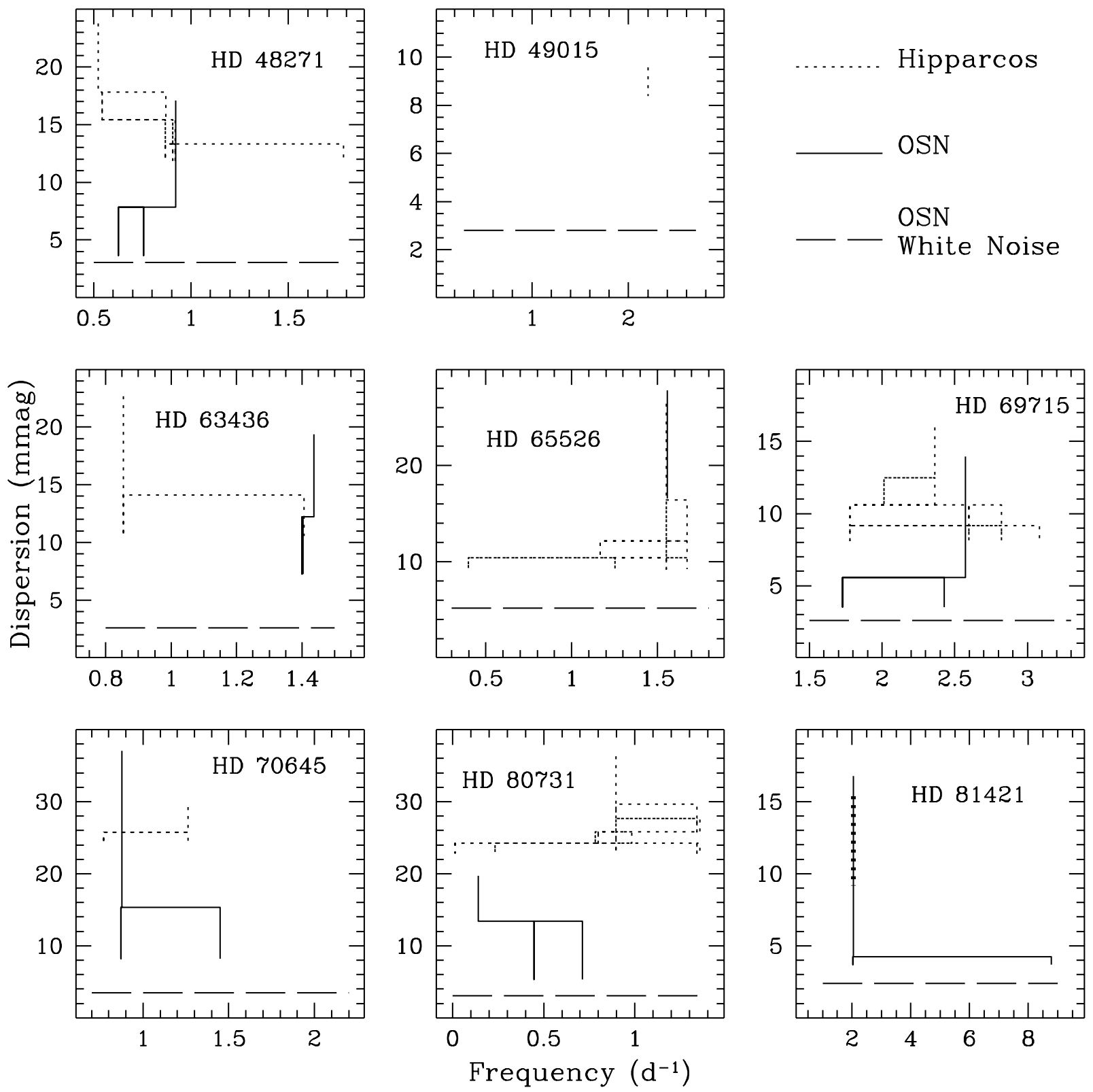

Fig. 3. Residual dispersion as a function of the fitting model for the different light curves: the dotted diagrams refer to Hipparcos $V$ series, the solid ones concern our Sierra Nevada $v$ data whereas the dashed horizontal lines represent the corresponding white noise levels. The height of each multiplet expresses the dispersion gain obtained through the corresponding frequency model. In the case of HD 48271, for example (top panel on the left), the 23.76 mmag standard deviation of the Hipparcos photometry is converted in a 17.81 mmag residual dispersion by a monoperiodic model $\left(f=0.52436 \mathrm{~cd}^{-1}\right)$ while with two frequencies $\left(0.54463\right.$ and $\left.0.87183 \mathrm{~cd}^{-1}\right)$ the residual standard deviation lowers to $15.40 \mathrm{mmag}$, and so on. The solid diagram presented in the same panel shows how the $17.07 \mathrm{mmag}$ dispersion of the Sierra Nevada $v$ light curve is reduced to $7.70 \mathrm{mmag}$ by our monoperiodic model $\left(f=0.918 \mathrm{~cd}^{-1}\right)$ and to $3.74 \mathrm{mmag}$ (close to the white noise level of $3.07 \mathrm{mmag}$ indicated by the dashed line) by the corresponding two frequency model presented in Table $5\left(0.623+0.750 \mathrm{~cd}^{-1}\right)$.

are known to lay in a such restricted area of the HR diagram. Nevertheless monoperiodic $\gamma$ Doradus stars exist, but at a level in which only photometry is available, albeit multicolour, no more than a flag for further studies can be put on the monoperiodic cases.

To assess the multiperiodic character of a star we do not necessarily need a coincidence of the frequency values found in the two data sets on the same data set. We consider a star multiperiodic if in each of the data set available and each of the analyses performed there is a clear need for more than one frequency to model the curve. Were the data optimally sampled and homogeneous in temporal baseline and distribution we would expect coincidence even in the frequency values obtained. However, in variable stars of this type, amplitudes and also frequencies are known to be changeable in short time scales (see Zerbi \& Kaye 2002 for details) and both the epoch and the baseline of the data compared in this paper are very different to pretend coherence even in the frequency values. 
Conversely in the case such a coherence shows up we can state with a certain level of confidence that such a frequency really corresponds to a physical oscillation.

As regards multiperiodicity the plots reported in Figs. 2 and 3 makes a convincing case of the multiperiodic character of six out of eight of the stars studied. The exceptions are HD 81421 and HD 49015. The former is clearly monoperiodic while the latter has been already anticipated as a puzzling case.

In the case of HD 49015 the Hipparcos data set the Multifre analysis stops at one-frequency model providing a value of $2.2050 \mathrm{~cd}^{-1}$. This value does not correspond to that reported by Henry \& Henry (2000) who suggested $2.8969 \mathrm{~cd}^{-1}$ as the most likely frequency between their aliases 1.8950 and $3.8986 \mathrm{~cd}^{-1}$. Curiously, however, analyzing the photometry collected by Henry \& Henry (2000) by means of Multifre we could confirm their results, i.e., a single frequency of $2.8964 \pm 0.0006 \mathrm{~cd}^{-1}$. A possible reason for the above discrepancy could be the high level of white noise $(S / N=1.3 \mathrm{mag})$ in the Hipparcos data of this star (see Fig. 3). Unfortunately, the OSN photometry does not provide any further help since the frequencies pointed out do not match with any of the previous values. Given that the results detected in both $v$ and $b$ filters are different between themselves, they are not shown in either of the figures. On the other hand, as we have commented before, considering that the epochs of the three data sets are totally different each frequency could have dominated during an observing campaign whereas both components are not detected in our photometry. But this argument is clearly not enough to confirm that this object is a $\gamma$ Doradus variable.

Previously to our observations, the star HD 81421 had only the frequency estimate reported in the Hipparcos Catalogue and it had also been object of other studies by Duerbeck (1997) and Eyer (2002) being classified as a contact binary (EW) but with no indication of a frequency estimate. The results obtained in our analysis are instead in good agreement with those of the Hipparcos data base. In both Hipparcos and OSN data only one frequency has been identified $\sim 2.04 \mathrm{~cd}^{-1}$. As stated above the monoperiodicity demands further investigation since the variability in this star could be due to causes different from oscillation, e.g. binarity of EW type. However color curves help in this case. In an eclipsing binary system, when the two stars have almost equal physical parameters, we do not expect any variations in the colour indices and hence in the amplitudes corresponding to the different filters. If instead the stars have appreciable differences in the physical parameters these variations exist but with a phase lag of 0 or $\pi$ between the lights curve and any colour calibrating the temperature. This does not happen if the variability is due to pulsation. The results of the Fourier fitting to our measurements are summarized in Table 6 with amplitudes, phases, mean values, data dispersion and residuals for the four $u v b y$ filters and $b-y$ index. Despite the high error bars, the phase difference calculated between $y$ and $b-y$ is of $\sim 1.37 \mathrm{rad}$. In addition the amplitudes in the different Strömgren filters show a progressive decrease of magnitude $(v, b, y$ and $u$ ) typically of A-F type pulsating stars. We are therefore prompted by our data to conclude that HD 81421 is not an EW binary system an hence likely a monoperiodic $\gamma$ Doradus variable. An external warning however comes for the identification of HD 81421 with an $\mathrm{X}$-ray source. The mechanism of generation of X-rays in close binary systems is well known while $\mathrm{X}$-ray emission from single early F-type stars is generally lacking or weak.

Multiperiodicity is clearly shown by the rest of program stars confirming their $\gamma$ Doradus nature. For HD 48271 our results confirm the frequencies proposed in previous analyses and even adds further modes. In fact the value detected in the OSN photometry, $0.918 \mathrm{~cd}^{-1}$, corresponds to the frequency with the largest amplitude in the Hipparcos solution. The frequency $1.783 \mathrm{~cd}^{-1}$ detected by Multifre can be interpreted as a coupling term (sum of 0.869 and $0.918 \mathrm{~cd}^{-1}$ ) making clear the existence of the both components.

Like other stars in the program, in the case of HD 65526 our analysis finds a higher number of components than Handler's method using the Hipparcos data. Taking into account our observations, the presence of the frequency $1.556 \mathrm{~cd}^{-1}$ is confirmed, which has a relationship 2:1 with the frequency $0.77641 \mathrm{~cd}^{-1}$ reported in the original Hipparcos Catalogue. In the case of HD 63436, marked as "unsolved" in the Hipparcos data base and suspected to have some signal around $1.4 \mathrm{~cd}^{-1}$ by Handler (1999), we could point out two excited frequencies, one of which in the Handler's range.

For HD 80731, the Multifre code confirms the existence of the main frequency $0.896 \mathrm{~cd}^{-1}$ in the Hipparcos data set. The $0.713 \mathrm{~cd}^{-1}$ component of our photometry is consistent with the possible Handler's frequency, $0.7 \mathrm{~cd}^{-1}$. Also, we find two components at $0.896 \mathrm{~cd}^{-1}$ and $1.342 \mathrm{~cd}^{-1}$ corresponding to the $2: 1$ and $3: 1$ ratios of the $0.447 \mathrm{~cd}$ term, frequency obtained independently from the OSN analysis.

In two cases, namely HD 69175 and HD 70645, multiperiodicity has been found in Hipparcos and OSN curves, stating their $\gamma$ Doradus nature, but the frequency value could not be directly compared to those proposed by other authors. In the case of HD 69715, applying the Multifre code to the Hipparcos photometry up to the two-frequency model, we find the same values than Handler (1999). However, when increasing the number of components, Multifre modified the values toward those obtained in the analysis of OSN data. In general, the circumstance that the same frequency is not repeated through the successive models is more frequent in the OSN results. Thus, we suspect that the drastic change in the frequency values produced in HD 69715 is more related to the poor spectral window than to the amount of data.

In the case of HD 70645 the first analysis we performed on the Hipparcos curves was biased by a few data-points which were spurious and out of scale. These points created a very high level of white noise at the point that Multifre code could not provide any solution. Only after elimination of the five measurements with largest dispersion has it been possible to obtain the frequencies presented in Table 5, where only the component $0.77076 \mathrm{~cd}^{-1}$ is in the error bar of the signal $0.8 \mathrm{~cd}^{-1}$ previously proposed as second component. Curiously, we could reproduce exactly the frequencies found by Handler (1999) by means of the prewhitening procedures and after removing three points of the initial Hipparcos data set. Thereby, since the result depends on the number of the measurements rejected in the data set, any frequency value pointed out from the Hipparcos photometry is 
Table 6. Results from Fourier analysis of OSN data on HD 81421. The phases have been computed with respect $T_{0}=2451916.5794$ HJD.

\begin{tabular}{lccccc}
\hline \hline \multicolumn{7}{c}{$\mathrm{HD} 81421\left(f=2.046 \pm 0.003 \mathrm{~cd}^{-1}\right)$} \\
\hline Colour & $\begin{array}{c}\text { A } \\
(\mathrm{mag})\end{array}$ & $\begin{array}{c}\varphi \\
\mathrm{rad})\end{array}$ & $\begin{array}{c}\text { Mean val. } \\
(\mathrm{mag})\end{array}$ & $\begin{array}{c}\text { Data disp. } \\
(\mathrm{mag})\end{array}$ & $\begin{array}{c}\text { Residuals } \\
(\mathrm{mag})\end{array}$ \\
\hline$u$ & $0.0188 \pm 0.0009$ & $0.50 \pm 0.11$ & $-0.3557 \pm 0.0015$ & 0.0161 & 0.0088 \\
$v$ & $0.0225 \pm 0.0010$ & $0.43 \pm 0.04$ & $-0.2543 \pm 0.0007$ & 0.0166 & 0.0041 \\
$b$ & $0.0204 \pm 0.0009$ & $0.40 \pm 0.04$ & $-0.2851 \pm 0.0007$ & 0.0150 & 0.0038 \\
$y$ & $0.0196 \pm 0.0011$ & $0.26 \pm 0.06$ & $-0.3240 \pm 0.0008$ & 0.0143 & 0.0047 \\
$b-y$ & $0.0030 \pm 0.0021$ & $1.63 \pm 0.30$ & $0.0386 \pm 0.0007$ & 0.0041 & 0.0037 \\
\hline
\end{tabular}

highly uncertain. However, the frequency at $0.877 \mathrm{~cd}^{-1}$ have been detected in the analysis of the OSN data set only barely similar to the $0.8 \mathrm{~cd}^{-1}$ proposed by Handler as second component. Albeit the unknown frequency values HD 70645 is clearly multiperiodic and hence a $\gamma$ Doradus star.

\section{Conclusions}

We presented in this paper the results of new uvby photometry of eight $\gamma$ Doradus candidates. Also a comparative analysis with a independent and different data set, Hipparcos photometry, has been performed using the new code of period-search Multifre. When possible, we have included for comparison and discussion the results of the frequency analyses previously published by other authors. In sight of the presented results, the code used Multifre provided evidence to be a powerful periodsearch tool. Wherever its outcomes differed from the ones of more classical methods used by other authors in the analysis of the Hipparcos photometry, the corresponding model proved to fit the data better than the preceding descriptions.

Following the above guideline we could confirm that all program objects, with the exception of HD 49015, are $\gamma$ Doradus stars. We cannot either confirm or reject the $\gamma$ Dor nature of HD 49015. In this case, the single frequency detected in the Hipparcos data is not consistent with the variation found by Henry \& Henry (2000) in their light curve and there is no evidence of such frequencies in our observations. Perhaps to understand better these results, particular characteristics of this type of stars as amplitude and frequency variations in short time scales must be taking into account.

In spite of its monoperiodic nature, we present arguments to state that HD 82421 is also a $\gamma$ Doradus variable. Our analysis of the both Hipparcos and OSN data sets are consistent with the single frequency reported by the Hipparcos Catalogue. The rest of the observed objects show multiperiocity independently of the data set.

Although the number of measurements collected in Sierra Nevada is relatively small, they allowed to independently confirm some of the frequencies found in the Hipparcos data set. Indeed, we can consider $f=0.918 \mathrm{~cd}^{-1}$ (HD 48271), $f=1.40 \mathrm{~cd}^{-1}$ (HD 63436), $f=1.556 \mathrm{~cd}^{-1}$ (HD 65526), $f=0.896 \mathrm{~cd}^{-1}$ (HD 80731) and $f=2.046 \mathrm{~cd}^{-1}$ (HD 81421) as physically meaningful.

Acknowledgements. We are grateful to Eloy Rodríguez who computed and provided some useful physical parameters of the stars.
We thank the staff of the Observatorio de Sierra Nevada (Instituto de Astrofísica de Andalucía (CSIC)) for the assistance during the observations. Part of this work was supported by the Osservatorio Astronomnico di Brera and by the Agenzia Spaziale Italiana (ASI Contract I/R/ 037/01). We also acknowledge the financial support by a Marie Curie Fellowship of the European Community programme Improving the Human Potential under contract number HPMF-CT2001-01146. This research has made use of the Simbad data base, operated at CDS, Strasbourg, France.

\section{References}

Aerts, C., Eyer, L., \& Kestens, E. 1998, A\&A, 337, 790

Arentoft, T., Sterken, C., \& Knudse, M. 2001, A\&A, 380, 599

Baglin, A., \& Foing, B. H. 1999, Adv. Space Res., 24, 245

Balona, L. A., Bohm, T., Foing, B. H., et al. 1996, MNRAS, 281, 1315

Breger, M., Handler, G., Garrido, R., et al. 1997, A\&A, 324, 566

Bossi, M., \& Nuñez, N. S. 2002, in preparation

Duerbeck, H. W. 1997, Inf. Bull. Variable Stars, 4513

ESA 1997, The Hipparcos Catalogue, ESA SP-1200

Eyer, L., \& Aerts, C. 2000, A\&A, 361, 201

Eyer, L., Aerts, C., van Loon, M., Bouckaert, F., \& Cuypers, J. 2002, in Observational aspects of Pulsating B \& A Stars, ed. C. Sterken, \& D. W. Kurtz, ASP Conf. Ser., 256, 203

Fleming, T. A., Molendi, S., Maccacaro, T., \& Wolter, A. 1995, ApJS, 99,701

Fukuda, I. 1982, PASP, 94, 271

Guzik, J. A., Kaye, A. B., Bradley, P. A., Cox, A. N., \& Neuforge, C. 2000, ApJ, 542, L57

Handler, G. 1999, MNRAS, 309, L19

Handler, G., \& Kaye, A. B. 2000, http://sirius.saao.ac.za/ gerald/gdorlist.html

Hauck, B., \& Mermilliod, M. 1998, A\&AS, 129, 431

Henry, G. W., \& Henry, S. M. 2000, Inf. Bull. Variable Stars, 4899

Kaye, A. B., \& Zerbi, F. M. 1997, Delta Scuti Star Newsletter, 11, 32

Kaye, A. B., Handler, G., Krisciunas, K., Poretti, E., \& Zerbi, F. M. 1999, PASP, 111, 840

Kjeldsen, H., Bedding, T. R., \& Christensen-Dalsgaard, J. 2000, in The impact of Large-scale Surveys on Pulsating Star Research, ed. L. Szabados, \& D. W. Kurtz, ASP Conf. Ser., 203, 73

Kim, S.-L., Chun, M.-Y., Park, B.-G., et al. 2001, A\&A, 371, 571

Martín, S. 2002, in ASP Conf. Ser., Interplay between periodic, cyclic and stochastic variability in selected areas of the HR Diagram, ed. C. Sterken, in press

Martín, S., \& Rodríguez, E. 2000, A\&A, 358, 287

Matthews, J. M., Kuschning, R., Walker, G. A. H., et al. 2000, in The impact of Large-scale Surveys on Pulsating Star Research, ed. L. Szabados, \& D. W. Kurtz, ASP Conf. Ser., 203, 74 
Micela, G., Favata, F., \& Sciortino, S. 1997, Hipparcos Venice'97, ESA SP-402, 391

Nissen, P. E. 1988, A\&A, 199, 146

Nordström, B., Stefanik, R. P., Latham, D. W., \& Andersen, J. 1997, A\&A, 126, 21

Philip, A. G. D., \& Egret, D. 1980, A\&A, 40, 199

Philip, A. G. D., Miller, T. M., \& Relyea, L. J. 1976, Dudley Obs. Rep., 12,1

Rodríguez, E., \& Breger, M. 2001, A\&A, 366, 178

Rodríguez, E., González-Bedolla, S. F., Rolland, A., Costa, V., \& López de Coca, P. 1997, A\&A, 324, 959

Smalley, B. 1993, A\&A, 274, 391
Smalley, B., \& Kupka, F. 1997, A\&A, 328, 349

Turon, C., Crézé, M., Egret, D., et al. 1992, ESA SP-1136, vols. I-VII

Zerbi, F. M. 2000, in Delta Scuti and Related Stars, ed. M. Breger, \& M. H. Montgomery, ASP Conf. Ser., 210, 332

Zerbi, F. M., \& Kaye, A. B. 2002, in Radial and Nonradial Pulsations as Probes of Stellar Physics, ed. C. Aerts, T. R. Bedding, \& J. Christensen-Dalsgaard, ASP Conf. Ser., 259, 494

Zerbi, F. M., Mantegazza, L., Campana, S., \& Antonello, E. 1998, PASP, 110,804

Zerbi, F. M., Rodríguez, E., Garrido, R., et al. 1999, MNRAS, 303, 275 